\title{
Sugars and fat - a healthy way to generate functional regulatory $\mathrm{T}$ cells
}

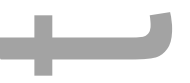

Sarah Sharon Gabriel ${ }^{1,2} \&$ Axel Kallies ${ }^{1,2}$

प1

${ }^{1}$ The Walter and Eliza Hall Institute of Medical Research, 1G Royal Parade, Parkville, Victoria, 3052, Australia.

${ }^{2}$ The Department of Medical Biology, University of Melbourne, Parkville, Victoria, 3010, Australia.
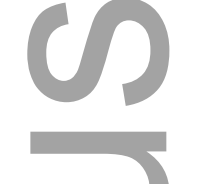

Correspondence should be addressed to A.K. (kallies@wehi.edu.au).

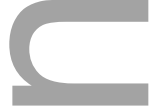

Abstract. Cellular metabolism has emerged as an important regulator of adaptive immunity. While the metabolic requirements of conventional T cells are increasingly understood, the role of cellular metabolism in Foxp3+ regulatory $\mathrm{T}$ (Treg) cells is less clear. Although it is well accepted that repression of Akt/mTOR, HIF-1 $\alpha$ and aerobic glycolysis are important for the efficient generation of Treg cells in vitro, clear evidence how these pathways impact on Treg-cell development in vivo is limited. Furthermore, newer studies have shown that the same pathways that appear to suppress Treg-cell development are active in and required for functionally mature Treg cells. Thus, it becomes increasingly evident that development and function of regulatory $T$ cells require different wiring of metabolic pathways. Finally, it is likely that critical differences remain to be uncovered between the metabolism of resting or 'naïve' Treg cells and those fully differentiated and actively engaged in suppression. In this comment, we briefly discuss our current understanding of Treg-cell metabolism and the need to address this area with new approaches based on in vivo models.

mTOR activity balances Treg-cell development and function. This commentary briefly discuss our current understanding of Treg-cell metabolism and the need to address this area with new approaches based on in vivo models.

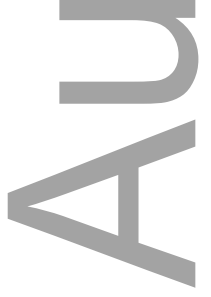

This is the author manuscript accepted for publication and has undergone full peer review but has not been through the copyediting, typesetting, pagination and proofreading process, which may lead to differences between this version and the Version of Record. Please cite this article as doi: 10.1002/eji.201646663.

This article is protected by copyright. All rights reserved. 

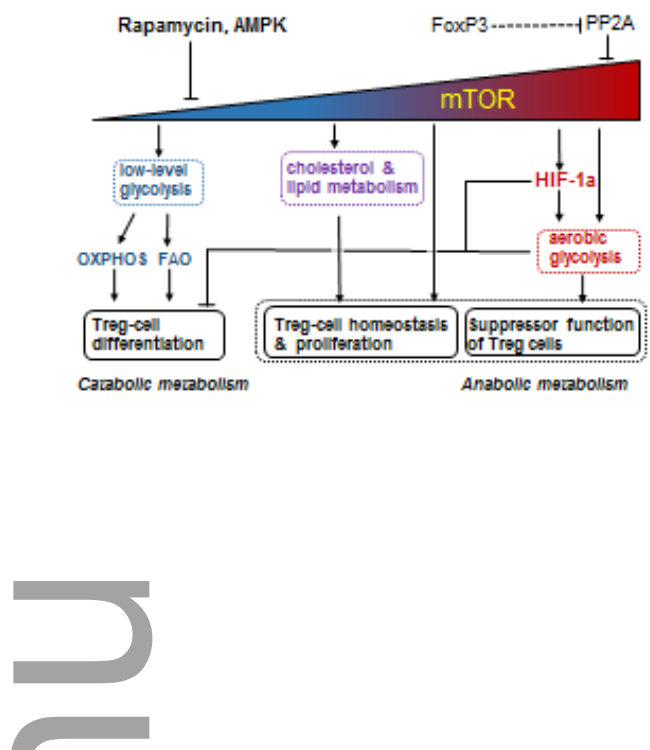

T-cell differentiation, function and cellular metabolism are tightly coordinated and multiple pathways link the molecular control of both processes. As T cells differentiate into effector or memory cells in response to antigen-receptor stimulation and cytokines, their cellular metabolism undergoes profound changes in order to adapt to novel requirements. However, at the same time non-immunological cues such as availability of nutrients, oxygen tension or the accumulation of metabolites influence cellular metabolism and thereby affect the activity and fate of T cells.

Naïve T cells display low energy demand and derive their energy mainly through the highly efficient process of oxidative phosphorylation (OXPHOS). Low-level glycolytic activity yields pyruvate, which is imported into the mitochondria and metabolized in the tricarboxylic acid (TCA) cycle, together with other substrates such as fatty acids (FA) via $\beta$-oxidation (also called fatty acid oxidation FAO). Ultimately, the reducing equivalents produced in the TCA cycle fuel ATP production via OXPHOS (1). Upon activation, T-cell metabolism undergoes dramatic changes in order to meet the high energy demands associated with the proliferative burst and effector molecule production. Similar to highly proliferative cancer cells, activated T cells switch to aerobic glycolysis, which is less efficient for ATP production, but generates precursor molecules for the generation of lipids, amino acids and nucleotides needed for rapid proliferation (1).

T-cell metabolism is coordinated by a complex network of conserved signaling pathways involving the mammalian target of rapamycin (mTOR), AMP-activated protein kinase (AMPK) and the transcription factors hypoxia-inducible factor $1 \alpha$ (HIF-1 $\alpha$ ) and MYC. mTOR is activated rapidly in response to T-cell receptor (TCR) ligation and co-stimulation and its activity is further modulated by metabolic and environmental cues, such as glucose and essential amino acid availability or growth factors (2). Once activated, mTOR plays a central role in rewiring cellular metabolism towards aerobic glycolysis and in promoting cell growth, proliferation and migration $(2,3)$ (Figure 1). mTOR exists in the form of two protein complexes, mTORC1 and mTORC1, with mTORC1 being the main target of rapamycin. HIF-1 $\alpha$ and MYC are activated in response to TCR stimulation, and promote the

This article is protected by copyright. All rights reserved. 
expression of genes needed for efficient glycolysis $(4,5)$. In contrast, AMPK is activated in response to stress and decreased cellular ATP levels and promotes pathways that conserve energy. As such, AMPK counteracts anabolic pathways in activated T cells such as FA synthesis by inhibiting mTORC1 activity, and instead promotes energy-efficient ATP production through OXPHOS and mitochondrial FA oxidation (5) (Figure 1).

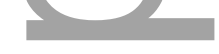

Treg cells are a specialized lineage of $\mathrm{CD}^{+} \mathrm{T}$ cells that can efficiently suppress immune responses to self and foreign antigen. Treg cells also fulfil critical functions in tissue repair, homeostasis and limiting metabolic diseases. Functional impairments of Treg cells have been shown to result in autoimmunity, and the loss of Treg cells leads to fatal immunopathology in both humans and mice $(6,7)$. The vast majority of Treg cells develop in the thymus in a TCR- and IL-2-dependent manner. A smaller proportion of Treg cells is induced in the periphery, in particular in the gastrointestinal tract, in response to microbiota-derived antigens and metabolites, such as short-chain FA (8). Based on a large body of evidence, Treg cells are believed to display very distinct metabolic requirements compared to their effector T-cell counterparts. Analysis of in vitro polarized $\mathrm{CD} 4^{+}$cells suggested that while Akt/mTOR- and HIF-1 $\alpha$-mediated aerobic glycolysis is crucial for $T$ helper (Th)-cell differentiation, Treg cells rely largely on AMPK-promoted mitochondrial FAO (9-11). This model is supported by various studies showing that genetic or pharmacological interventions that modulate either metabolic arm affect Th-cell and Treg-cell differentiation in an opposing fashion. In particular, blockade of FAO was shown to preclude the generation of Treg cells, while stimulation of this pathway, either by adding exogenous FA or by pharmacological activation of AMPK, promoted Tregcell generation (9). On the other hand, inhibition of glycolysis resulted in preferential Treg-cell generation, as shown by the pharmacological blockade of mTOR activity, or by the genetic deletion of mTOR or HIF-1 $\alpha(9,10,12)$. In contrast, constitutive active Akt/mTOR signaling promoted Th-cell generation (11). Interestingly, FA metabolism appears to differently regulated between Th cells and Treg cells; for example, de novo FA synthesis is only required for Th-cell but not Treg-cell differentiation (13). Together, these results have created a paradigm according to which the activation of anabolic pathways linked to aerobic glycolysis is crucial for successful CD $4^{+}$effector cell differentiation and expansion, whereas the same pathways seem to be redundant or even inhibitory for Treg-cell differentiation.

However, an important caveat of the aforementioned studies is the fact that the vast majority of findings were derived from in vitro experiments, where naïve $\mathrm{CD} 4^{+}$cells were stimulated under Th- or Treg-polarizing conditions. This raises the question of: To what degree do these findings represent the in vivo reality? Do in vitro-induced (i)Treg cells correctly represent the metabolic phenotypes and requirements of Treg cells that occur in vivo? Do thymically derived $(\mathrm{t})$ Treg cells and peripherally induced $(p)$ Treg cells show differences in their utilization of metabolic pathways? During in vitro iTreg-cell differentiation, cells are routinely exposed to high concentrations of TGF- $\beta$, which is known to be involved in regulating various biological processes, including cell growth, proliferation and cellular metabolism by inhibiting the Akt/mTOR axis $(12,14,15)$. Thus, the dampened cellular metabolism observed in iTreg cells might be an indirect consequence prolonged TGF- $\beta$ exposure rather than a requirement for iTreg-cell generation. Notably, genetic loss of mTOR results in hyperactivation of Smad3, which is downstream of TGF- $\beta$ signaling (12) and required for iTreg-cell

This article is protected by copyright. All rights reserved. 
induction $(14,16)$. Thus, increased Treg-cell generation in response to mTOR blockade may also be a consequence of ectopic activation of Smad3, rather than due to a reduction in aerobic glycolysis. However, so far it has not been stringently tested whether dampening of the Akt/mTOR axis is critical for the in vivo generation of pTreg cells. Similarly, it is unclear which metabolic pathways are essential for the thymic generation of Treg cells, although one study has suggested that receptor S1P1-mediated activation of mTOR plays a limiting role (17). In this context, a FoxP3 double-reporter mouse, which allows the discrimination of thymically and peripherally derived Treg cells, might prove to be a useful tool (18). The basis for this mouse model is the observation that a Foxp3-green fluorescence (GFP) transgene (19) is only activated during thymic Treg-cell development, but not during differentiation of Foxp $3^{+}$Treg cells in vitro or in the periphery of mice. Thus, combination of the Foxp3-GFP transgene with a Foxp3-red fluorescence (RFP) knock-in allele (20) that faithfully reports Foxp3 expression in both tTreg and pTreg cells, results in a Foxp3 double reporter, in which pTreg cells are RFP+GFP-, while tTreg cells are positive for both RFP and GFP (18). Notably, the authors show that other molecules currently considered to be expressed specifically on thymically derived Treg cells, including Nrlp1 $(21,22)$ and Helios (8), were expressed similarly in Foxp3 ${ }^{+} \mathrm{GFP}^{-}$ and $\mathrm{GFP}^{+}$Treg cells (18), suggesting that these surrogate markers of tTreg cells should be treated with caution. However, additional experiments should be performed to fully characterize the double reporter mouse model, including a detailed analysis of the colonic Treg-cell compartment. Furthermore, it should be attempted to reconcile the apparent lack of correlation between the phenotypes of pTreg identified based on Nrlp1 or Helios with those identified using the Foxp3 double reporter. Nevertheless, new mouse models and experimental strategies are required to thoroughly dissect the impact of metabolic pathways on Treg-cell development both in the thymus and in the periphery.

In addition to the limitations of our current understanding of the metabolic requirements of Treg-cell development discussed above, there are other reasons why the prevailing model of a 'metabolic Treg-Th cell dichotomy' should be revisited. Recent studies analyzing the metabolic profile of naturally occurring Treg cells directly after ex vivo isolation are difficult to reconcile with the idea that Treg cells do not depend on aerobic glycolysis. For example, cycling Treg cells display increased Glut1 expression and activation of mTORC1 (23), and in comparison to any other cell type, including tumor-infiltrating $\mathrm{CD}^{+} \mathrm{T}$ cells, splenic and intratumoral Treg cells display higher glycolytic activity (24), a hallmark of proliferating cells. Indeed, Treg cells consistently display high turnover, probably in response to self-antigen stimulation (25-27). These findings are in line with a comprehensive study comparing the metabolic profiles of ex vivo isolated human Treg cells and conventional T cells. At steady state, Treg cells showed increased activity of mTOR and expression of proteins associated with the glycolytic pathway and lipid synthesis, a significantly higher glycolytic flux, but reduced expression of components of the TCA cycle as compared to conventional T cells $(26,28)$ (Figure 1). Furthermore, several studies indicate that important promotors of glycolytic activity are needed for Treg-cell suppressive function in vivo. For example, Treg cells lacking HIF-1 $\alpha$ or mTORC1 fail to control T-cell-mediated colitis $(29,30)$. mTORC1 signaling has been shown to be constitutively active in Treg cells and to be required for proliferation and suppressive function, as genetic loss of mTORC1 in specifically in Treg cells resulted in fatal autoimmune disease similar to the scurfy phenotype (30) (Figure 1). Specifically, mTORC1 was found to be essential for Treg-cell homeostasis and suppressive function via promotion of lipid and cholesterol biosynthesis from glucose (30) (Figure 1). On the other side, unrestrained Akt/mTOR activity, due to loss of negative 
regulators such PP2A and PTEN, has been shown to impair Treg-cell stability and suppressive function, suggesting that close control of mTOR activity in vivo is required $(31,32)$. Thus, in vivo studies reveal the importance of metabolic regulators in Treg cells, which previously were thought to be negligible in in vitro-generated Treg cells.

When analyzing metabolism of Treg cells, it is important to note that in vivo-occurring Treg cells do not represent a homogenous population. In addition to the above-mentioned discrimination of Treg cells based on their developmental origin, Treg cells display different activation states, which are likely to result in different metabolic activity. Broadly, Treg cells can be divided into central (c)Treg cells, which mainly recirculate through lymphoid tissues and show a naïve phenotype, and effector (e)Treg cells, which accumulate in non-lymphoid tissues and display high expression of activation markers and the transcription factor Blimp-1. Most importantly, eTreg cells show superior suppressive activity, and secretion of the immune suppressive cytokine IL-10 is limited to eTreg cells $(33,34)$. Notably, eTreg cells can functionally adapt to their local environment to exert tissue-specific functions (35). For example, Treg cells found in the visceral adipose tissue express the transcription factor PPAR $\gamma$, which induces an increased expression of genes associated with lipid metabolism, such as enzymes for FA synthesis, oxidation and transport (36). It is technically challenging to characterize the metabolism of various Treg-cell subsets ex vivo, as these cells are rare and their isolation requires a combination of surface markers and reporters for lineage-defining transcription factors. In this regard, a Blimp-1/Foxp3 double-reporter mouse that allows isolation of eTreg-cell subsets may provide a useful tool (37). Thus, when examining the impact of metabolic pathways on Treg-cell biology, it is essential to consider the different origin and activation status of Treg-cell populations and their effector counterpart.

Finally, a detailed understanding of the molecular differences between Treg cells and their conventional counterparts is required to fully unravel Treg-cell-specific metabolic requirements. For example, it has been shown by Dimeloe et al. that various $\mathrm{CD}^{+} \mathrm{T}$-cell populations express different amounts of the multidrug resistance protein $A B C B 1$, also called P-glycoprotein, encoded by multidrug resistant gene 1 (38). While Treg cells appear to lack expression of $A B C B 1$, the bulk of $\mathrm{CD}^{+}$and $\mathrm{CD} 8^{+} \mathrm{T}$ cells, including central and effector memory cells, express this efflux pump $(38,39)$. Notably, mitotracker green, which is a commonly used stain to determine mitochondrial mass, is a substrate for $A B C B 1$. Therefore, caution is required when interpreting results based on mitochondrial stains in various T cells subsets and in particular in Treg cells.

In summary, new approaches are required to unravel the metabolic regulation of Treg-cell development and function. These approaches need to take into consideration the potential differences in metabolism in Treg-cell subpopulations based on their developmental origin as well as the distinct maturation stages of Treg cells in the periphery. Thorough understanding the metabolism of Treg cells offer novel pharmacological targets for the specific promotion or inhibition of Treg-cell development and function. In order to meet this goal, we have to combine our advanced understanding of in vivo Treg-cell biology with the field of immunometabolism.

This article is protected by copyright. All rights reserved. 
Acknowledgments. We wish to thank the members of the Kallies lab for discussion. This work was supported by fellowships from the Sylvia and Charles Viertel Foundation (Senior Medical Research Fellowship to AK) and the Swiss National Science Foundation (Postdoctoral fellowship to SG).

Conflict of interest. The authors declare no financial or commercial conflict of interest.

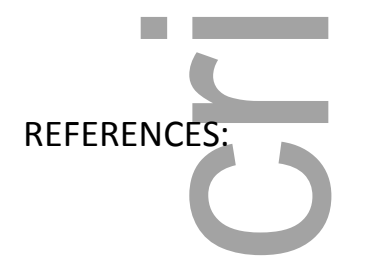

1. Buck MD, O'Sullivan D, Pearce EL. T cell metabolism drives immunity. J Exp Med. 2015;212(9):1345-60.

2. Chi H. Regulation and function of mTOR signalling in T cell fate decisions. Nat Rev Immunol. 2012;12(5):325-38.

3. Pollizzi KN, Powell JD. Integrating canonical and metabolic signalling programmes in the regulation of T cell responses. Nat Rev Immunol. 2014;14(7):435-46.

4. Wang R, Dillon CP, Shi LZ, Milasta S, Carter R, Finkelstein D, et al. The transcription factor Myc controls metabolic reprogramming upon T lymphocyte activation. Immunity. 2011;35(6):87182.

5. Johnson MO, Siska PJ, Contreras DC, Rathmell JC. Nutrients and the microenvironment to feed a T cell army. Semin Immunol. 2016.

6. Burzyn D, Benoist C, Mathis D. Regulatory T cells in nonlymphoid tissues. Nat Immunol. 2013;14(10):1007-13.

7. Ohkura N, Kitagawa Y, Sakaguchi S. Development and maintenance of regulatory T cells. Immunity. 2013;38(3):414-23.

8. Tanoue T, Atarashi K, Honda K. Development and maintenance of intestinal regulatory $\mathrm{T}$ cells. Nat Rev Immunol. 2016;16(5):295-309.

9. Michalek RD, Gerriets VA, Jacobs SR, Macintyre AN, Maclver NJ, Mason EF, et al. Cutting edge: distinct glycolytic and lipid oxidative metabolic programs are essential for effector and regulatory CD4+ T cell subsets. J Immunol. 2011;186(6):3299-303.

10. Shi LZ, Wang R, Huang G, Vogel P, Neale G, Green DR, et al. HIF1alpha-dependent glycolytic pathway orchestrates a metabolic checkpoint for the differentiation of TH17 and Treg cells. J Exp Med. 2011;208(7):1367-76.

11. Haxhinasto $S$, Mathis $D$, Benoist $C$. The AKT-mTOR axis regulates de novo differentiation of CD4+Foxp3+ cells. J Exp Med. 2008;205(3):565-74.

12. Delgoffe GM, Kole TP, Zheng Y, Zarek PE, Matthews KL, Xiao B, et al. The mTOR kinase differentially regulates effector and regulatory $T$ cell lineage commitment. Immunity.

2009;30(6):832-44.

13. Berod L, Friedrich C, Nandan A, Freitag J, Hagemann S, Harmrolfs K, et al. De novo fatty acid synthesis controls the fate between regulatory T and T helper 17 cells. Nat Med. 2014;20(11):132733.

This article is protected by copyright. All rights reserved. 
14. Martinez GJ, Zhang Z, Chung Y, Reynolds JM, Lin X, Jetten AM, et al. Smad3 differentially regulates the induction of regulatory and inflammatory $T$ cell differentiation. J Biol Chem. 2009;284(51):35283-6.

15. Delisle JS, Giroux M, Boucher G, Landry JR, Hardy MP, Lemieux S, et al. The TGF-beta-Smad3 pathway inhibits CD28-dependent cell growth and proliferation of CD4 T cells. Genes Immun. 2013;14(2):115-26.

16. Li L, Iwamoto Y, Berezovskaya A, Boussiotis VA. A pathway regulated by cell cycle inhibitor p27Kip1 and checkpoint inhibitor Smad3 is involved in the induction of T cell tolerance. Nat Immunol. 2006;7(11):1157-65.

17. Liu G, Burns S, Huang G, Boyd K, Proia RL, Flavell RA, et al. The receptor S1P1 overrides regulatory T cell-mediated immune suppression through Akt-mTOR. Nat Immunol. 2009;10(7):769-

77.

18. Petzold C, Steinbronn N, Gereke M, Strasser RH, Sparwasser T, Bruder D, et al. Fluorochrome-based definition of naturally occurring Foxp3(+) regulatory $T$ cells of intra- and extrathymic origin. Eur J Immunol. 2014;44(12):3632-45.

19. Zhou X, Jeker LT, Fife BT, Zhu S, Anderson MS, McManus MT, et al. Selective miRNA disruption in T reg cells leads to uncontrolled autoimmunity. J Exp Med. 2008;205(9):1983-91. 20. Wan YY, Flavell RA. Identifying Foxp3-expressing suppressor T cells with a bicistronic reporter. Proc Natl Acad Sci U S A. 2005;102(14):5126-31.

21. Weiss JM, Bilate AM, Gobert M, Ding Y, Curotto de Lafaille MA, Parkhurst CN, et al. Neuropilin 1 is expressed on thymus-derived natural regulatory T cells, but not mucosa-generated induced Foxp3+ T reg cells. J Exp Med. 2012;209(10):1723-42, S1.

22. Yadav M, Louvet C, Davini D, Gardner JM, Martinez-Llordella M, Bailey-Bucktrout S, et al. Neuropilin-1 distinguishes natural and inducible regulatory $T$ cells among regulatory $T$ cell subsets in vivo. J Exp Med. 2012;209(10):1713-22, S1-19.

23. Gerriets VA, Kishton RJ, Johnson MO, Cohen S, Siska PJ, Nichols AG, et al. Foxp3 and Toll-like receptor signaling balance Treg cell anabolic metabolism for suppression. Nat Immunol. 2016.

24. Ho PC, Bihuniak JD, Macintyre AN, Staron M, Liu X, Amezquita R, et al. Phosphoenolpyruvate Is a Metabolic Checkpoint of Anti-tumor T Cell Responses. Cell. 2015;162(6):1217-28.

25. Fisson S, Darrasse-Jeze G, Litvinova E, Septier F, Klatzmann D, Liblau R, et al. Continuous activation of autoreactive CD4+CD25+ regulatory T cells in the steady state. J Exp Med.

2003;198(5):737-46.

26. Procaccini C, Carbone F, Di Silvestre D, Brambilla F, De Rosa V, Galgani M, et al. The Proteomic Landscape of Human Ex Vivo Regulatory and Conventional T Cells Reveals Specific Metabolic Requirements. Immunity. 2016;44(2):406-21.

27. Pierson W, Cauwe B, Policheni A, Schlenner SM, Franckaert D, Berges J, et al. Antiapoptotic $\mathrm{Mcl}-1$ is critical for the survival and niche-filling capacity of Foxp3(+) regulatory T cells. Nat Immunol. 2013;14(9):959-65.

28. Procaccini C, De Rosa V, Galgani M, Abanni L, Cali G, Porcellini A, et al. An oscillatory switch in mTOR kinase activity sets regulatory T cell responsiveness. Immunity. 2010;33(6):929-41.

29. Clambey ET, McNamee EN, Westrich JA, Glover LE, Campbell EL, Jedlicka P, et al. Hypoxiainducible factor-1 alpha-dependent induction of FoxP3 drives regulatory T-cell abundance and function during inflammatory hypoxia of the mucosa. Proc Natl Acad Sci U S A. 2012;109(41):E278493.

This article is protected by copyright. All rights reserved. 
30. Zeng H, Yang K, Cloer C, Neale G, Vogel P, Chi H. mTORC1 couples immune signals and metabolic programming to establish T(reg)-cell function. Nature. 2013;499(7459):485-90.

31. Apostolidis SA, Rodriguez-Rodriguez N, Suarez-Fueyo A, Dioufa N, Ozcan E, Crispin JC, et al. Phosphatase PP2A is requisite for the function of regulatory T cells. Nat Immunol. 2016;17(5):556-

64.

32. Huynh A, DuPage M, Priyadharshini B, Sage PT, Quiros J, Borges CM, et al. Control of PI(3) kinase in Treg cells maintains homeostasis and lineage stability. Nat Immunol. 2015;16(2):188-96. 33. Teh PP, Vasanthakumar A, Kallies A. Development and Function of Effector Regulatory $T$ Cells. Prog Mol Biol Transl Sci. 2015;136:155-74.

34. Cretney E, Xin A, Shi W, Minnich M, Masson F, Miasari M, et al. The transcription factors Blimp-1 and IRF4 jointly control the differentiation and function of effector regulatory $T$ cells. Nat Immunol. 2011;12(4):304-11.

35. Panduro M, Benoist C, Mathis D. Tissue Tregs. Annu Rev Immunol. 2016;34:609-33.

36. Cipolletta D, Feuerer M, Li A, Kamei N, Lee J, Shoelson SE, et al. PPAR-gamma is a major driver of the accumulation and phenotype of adipose tissue Treg cells. Nature. 2012;486(7404):54953.

37. Vasanthakumar A, Moro K, Xin A, Liao Y, Gloury R, Kawamoto S, et al. The transcriptional regulators IRF4, BATF and IL-33 orchestrate development and maintenance of adipose tissueresident regulatory T cells. Nat Immunol. 2015;16(3):276-85.

38. Dimeloe S, Frick C, Fischer M, Gubser PM, Razik L, Bantug GR, et al. Human regulatory T cells lack the cyclophosphamide-extruding transporter $A B C B 1$ and are more susceptible to cyclophosphamide-induced apoptosis. Eur J Immunol. 2014;44(12):3614-20.

39. Drach D, Zhao S, Drach J, Mahadevia R, Gattringer C, Huber H, et al. Subpopulations of normal peripheral blood and bone marrow cells express a functional multidrug resistant phenotype. Blood. 1992;80(11):2729-34.

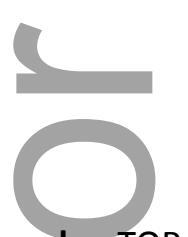

Figure Legend. mTOR activity balances Treg cell development and function.

Low mTOR activity translates into low-level glycolysis and preferential energy production through oxidative phosphorylation (OXPHOS) and fatty acid oxidation (FAO) in mitochondria. This metabolic state is associated with successful generation of Treg cells as demonstrated by increased Treg-cell development upon rapamycin treatment and AMPK activation. In contrast, high mTOR activity, HIF$1 \alpha$ and rewiring of cellular metabolism towards aerobic glycolysis inhibits Treg-cell generation but is required for suppressive function of Treg cells. mTOR also contributes to Treg-cell homeostasis and suppressive function via promotion of lipid and cholesterol biosynthesis from glucose. However, Treg-cell-specific restraint of mTOR activity via the phosphatase PP2A is required, as mTOR hyperactivation results in functional failure and Treg-cell instability.

This article is protected by copyright. All rights reserved. 


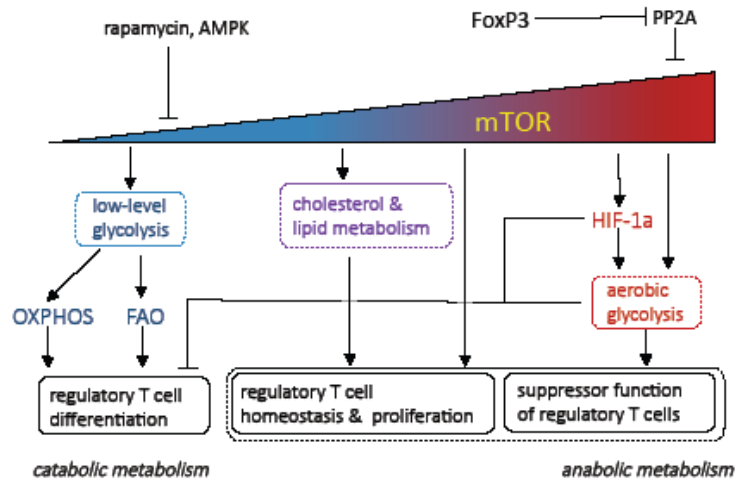

Gabriel \& Kallies, EJl comment

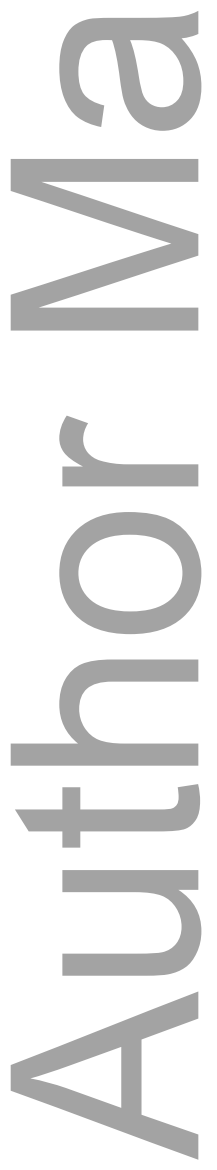

This article is protected by copyright. All rights reserved. 


\section{University Library}

\section{- M M N E R VA A gateway to Melbourne's research publications}

Minerva Access is the Institutional Repository of The University of Melbourne

Author/s:

Gabriel, SS;Kallies, A

Title:

Sugars and fat - A healthy way to generate functional regulatory $T$ cells

Date:

2016-12-01

Citation:

Gabriel, S. S. \& Kallies, A. (2016). Sugars and fat - A healthy way to generate functional regulatory T cells. EUROPEAN JOURNAL OF IMMUNOLOGY, 46 (12), pp.2705-2709. https://doi.org/10.1002/eji.201646663.

Persistent Link:

http://hdl.handle.net/11343/292200 